\title{
NÚCLEO DE ATENÇÃO FARMACÊUTICA DA UNIFAL-MG (NAFAU)
}

Caroline Gonçalves SIQUEIRA ${ }^{1}$

Marcela FORGERINI ${ }^{2}$

Ricardo Radighieri RASCADO ${ }^{3}$

Luciene Alves MARQUES ${ }^{4}$

\author{
${ }^{1}$ Graduanda em Farmácia pela Universidade Federal de Alfenas (UNIFAL-MG). ca_gsiqueira@hotmail.com \\ ${ }^{2}$ Graduada em Farmácia pela Universidade Federal de Alfenas (UNIFAL-MG). marcelinha_forgerini@hotmail.com \\ ${ }^{3}$ Doutor, Professor da Universidade Federal de Alfenas (UNIFAL-MG). ricardounifal@gmail.com \\ ${ }^{4}$ Doutora, Professora da Universidade Federal de Alfenas (UNIFAL-MG). lualvesmarques@gmail.com
}

Recebido em: 14/07/2016 - Aprovado em: 28/10/2016 - Disponibilizado em: 18/12/2016

\begin{abstract}
RESUMO:
Este artigo apresenta as atividades desenvolvidas e os resultados obtidos pelas ações do Núcleo de Atenção Farmacêutica da Universidade Federal de Alfenas (UNIFAL-MG) nos anos de 2012 a julho/2016. O Núcleo de Atenção Farmacêutica da UNIFAL é um componente extracurricular para o curso de Farmácia, indispensável à consolidação dos desempenhos profissionais desejados inerentes ao perfil do farmacêutico generalista. Com o farmacêutico atuando nas diversas vertentes da Atenção Farmacêutica, este projeto visa a interface entre pesquisa, ensino e extensão na área de atenção farmacêutica, incentivar o trabalho em equipe, promover atividades de ensino curricular e extracurricular, bem como orientar os pacientes quanto ao uso racional dos medicamentos a fim de promover a saúde dos mesmos e a visão que farmácia é um estabelecimento de saúde. O projeto realiza reuniões semanais com discussões de casos clínicos, campanhas educativas para a população e comunidade acadêmica com aferição de parâmetros bioquímicos e fisiológicos, palestras educativas, seminários, eventos científicos como o Simpósio Internacional de Atenção Farmacêutica, Estudos Dirigidos em Atenção Farmacêutica (EDAF), acompanhamento farmacoterapêutico de pacientes portadores de doenças crônicas. Ainda, disponibiliza ferramentas online - Blog (http://nafaumg.blogspot.com.br/) e o perfil no Facebook (NAFAU Atenção Farmacêutica), sendo estes atualizados com discussões da área.
\end{abstract}

Palavras-chave: Atenção farmacêutica. Acompanhamento farmacoterapêutico. Extensão universitária. Campanhas. População.

\begin{abstract}
:
This article presents the activities and results achieved by the actions of the Pharmaceutical Care Center of Federal University of Alfenas (UNIFAL-MG) in the years 2012 to July / 2016. The Pharmaceutical Care Center of UNIFAL is an extracurricular component to the course of Pharmacy, indispensable to the consolidation of the desired professional performance inherent to the general pharmaceutical profile. The pharmacist working in various aspects of pharmaceutical care, this project aims at the interface between research, teaching and extension in pharmaceutical care, to encourage teamwork, promote curricular and extracurricular school activities, and guide patients to use rational use of medicines in order to promote the health of themselves and the vision that pharmacy is a health facility. The project holds weekly meetings with the clinical case discussions, educational campaigns for the public and academic community with measurement of biochemical and physiological parameters, educational lectures, seminars, scientific events such as the International Symposium on Pharmaceutical Care, Directed Studies in Pharmaceutical Care (EDAF), pharmacotherapeutic monitoring of patients with chronic diseases. Also offers online tools - Blog (http://nafaumg.blogspot.com.br/) and Facebook page (NAFAU Pharmaceutical Care), these being updated with an area of discussions.
\end{abstract}

Keywords: Pharmaceutical care. pharmacotherapeutic monitoring. University Extension. Campaigns. Population. 


\section{INTRODUÇÃO}

A Atenção Farmacêutica conceitua-se como um modelo de prática farmacêutica, que compreende atitudes, valores éticos, comportamentos, habilidades, promoção e prevenção de doenças, e também a recuperação da saúde de forma integral. É a participação direta do farmacêutico com o paciente, visando o uso racional de medicamentos e a obtenção de resultados definidos e satisfatórios, com objetivo de obter melhoria na qualidade de vida e integralidade das ações de saúde do paciente (PEREIRA\& FREITAS, 2008).

Os problemas crescentes dos erros de medicação, correlacionado com a maior utilização dos medicamentos tem ocasionado impacto importante sobre a saúde das pessoas e sobre a economia dos sistemas de prestação de serviços. (FUNED, 2010).A atuação do farmacêutico inclui uma somatória de atitudes, comportamentos, responsabilidades e habilidades na prestação de serviços como a farmacoterapia, tendo como objetivo, alcançar resultados terapêuticos eficientes e seguros, privilegiando a saúde e a qualidade de vida do paciente (OLIVEIRA et al. 2005).

O acesso aos serviços de saúde é dependente da qualidade da atenção oferecida. Por isso, a estratégia da Atenção Farmacêutica privilegia o paciente e pretende colocar o usuário ao centro do sistema de prestação de serviços. Para garantir a efetividade das intervenções e a promoção do uso racional de medicamentos, está fortemente relacionada à colaboração do prescritor quanto do consumidor (BRASIL, 2010).

A filosofia da atenção farmacêutica afirma explicitamente a obrigação do profissional farmacêutico em assegurar para a sociedade o uso de medicação eficaz e segura. Esta obrigação é cumprida com o paciente, no momento em que o praticante aceita a responsabilidade de identificar, resolver e evitar problemas de cada doente na terapia medicamentosa. Essas responsabilidades são atendidas por meio do paradigma do cuidar de uma forma centrada ao paciente (STRAND et al., 2004).

O Núcleo de Atenção Farmacêutica da UNIFAL-MG é um programa de extensão universitária, e é um componente extracurricular para o curso de Farmácia, indispensável à consolidação do desempenho profissional desejado inerente ao perfil do farmacêutico generalista.

A extensão é um elo entre a Universidade, nas suas atividades de ensino e de pesquisa, com a sociedade e as demandas da população, possibilitando a formação do profissional humanista e cidadão, além de superar as desigualdades sociais existentes, com a multiplicação de informação e produção de conhecimento. 
Assim, tem-se hoje como princípio que, para a formação do profissional cidadão, é imprescindível uma interação efetiva com a sociedade (NOGUEIRA, 2000).

Durante o desenvolvimento de atividades de ensino, pesquisa e extensão, criam-se condições para que a formação acadêmica não restrinja aos aspectos técnicos e formais, contemplando aspectos sociais e políticos, promovendo a conscientização crítica (UFMG, 2016).

Assim, o Núcleo de Atenção Farmacêutica da UNIFAL (NAFAU) visa desenvolver atividades de extensão e pesquisa, e promover o ensino extracurricular através de cursos, palestras, oficinas; além de incentivar o trabalho em equipe e a interdisciplinaridade.

\section{OBJETIVO}

Este artigo tem por objetivo fazer um relato das experiências do grupo NAFAU desenvolvidas nos anos 2012 a julho de 2016.

\section{METODOLOGIA}

O NAFAU atualmente é composto por 18 discentes do curso de Farmácia, empenhados em realizar atividades na área de Atenção Farmacêutica. Foi fundado em 2012 por um acadêmico do curso e de 2012 até 2015, já teve 26 integrantes. O grupo está sob a coordenação de dois professores de Atenção Farmacêutica da UNIFAL-MG,

A equipe reúne-se semanalmente, com duração média de uma hora, para delinear as atividades que serão desenvolvidas.

O grupo desenvolveu ações de extensão: palestras educativas e minicursos no campus universitário com temas pertinentes e atualidades da área clínica para complementação da grade curricular dos graduandos, bem como troca de conhecimento e estímulo ao trabalho em equipe. Também foram realizadas Campanhas de Saúde no centro da cidade, feira livre de domingo, em supermercados e no campus universitário da UNIFAL-MG. Durante as campanhas foram realizadas aferições de parâmetros bioquímicos e fisiológicos, dentre eles: aferição de pressão arterial, glicemia capilar, colesterol total e triglicerídeos capilar, além de fornecer orientações quanto o uso racional de medicamentos.

O programa, neste ano de 2016, possui como ações registradas na Pró-Reitoria de Extensão, três projetos e um evento: 'Drogas: uma questão de escolha?', 'CIM Centro de Informação sobre Medicamentos', 'AtenFar no seu lar: acompanhamento farmacoterapêutico de pacientes' e, o evento: 'Setembro Amarelo'.

O projeto 'Drogas: uma questão de escolha?'possui por objetivo esclarecer e 
debater o tema das drogas com alunos do ensino médio e também com beneficiários do Centro de Referência de Assistência Social (CRAS) de dois bairros da cidade. O projeto visa abordar a prevenção do uso, abuso e dependência de drogas ilícitas, como também das lícitas que causam sérios problemas de saúde, como o álcool e cigarro.

$\mathrm{O}$ projeto CIM - Centro de Informação de Medicamentos tem por objetivo promover o uso racional de medicamentos por meio de informação técnico-científica objetiva, atualizada e devidamente processada para profissionais, pacientes e outros interessados em conhecer mais sobre os medicamentos.

No AtenFar, cada membro faz o seguimento farmacoterapêutico de um paciente, realizando o acompanhamento a domicílio, com aferições de glicemia capilar e pressão arterial, bem como anamnese farmacêutica (Método Dáder) para a identificação de problemas relacionados aos medicamentos (PRM) e de resultados clínicos negativos associados aos medicamentos (RNM), para que seja realizado a intervenção farmacêutica e esses problemas sejam resolvidos. $\mathrm{O}$ acompanhamento tem duração conforme a necessidade de cada paciente, sendo estes recrutados durante as campanhas de saúde realizadas pelo Núcleo.

Os projetos de pesquisa com interface com a extensão que estão sendo desenvolvidos durante o ano de 2016 são:a) Avaliação da síndrome de Burnout, resiliência e sintomas depressivos e ansiosos em bombeiros de Alfenas-MG; b) Elaboração de material educativo em saúde para portadores de deficiência visual e intervenções farmacêuticas em saúde; c) Avaliação da autoestima e da resiliência de funcionários terceirizados da Universidade Federal de Alfenas - Unifal-MG. Entre os projetos já concluídos encontram-se: a) Avaliação da efetividade da atenção farmacêutica no tratamento de pacientes com transtorno bipolar do humor; b) Efetividade da atenção farmacêutica sobre a qualidade de vida e resiliência de pacientes com depressão; c) Atenção Farmacêutica em pacientes ambulatoriais com depressão tratados com psicotrópicos.

\section{RESULTADOS E DISCUSSÃO}

Desde a criação, participaram do NAFAU 48 acadêmicos do curso de Farmácia, empenhados em realizar atividades de pesquisa, ensino e extensão, assim como proposto na metodologia do programa. Considerando que estas são complementares a dinâmica curricular do curso, há o aperfeiçoamento do acadêmico participante resultando no crescimento profissional e acadêmico dos envolvidos. O grupo estimula também o acadêmico a ter um pensamento crítico-reflexivo sobre 
diversos temas e assuntos da atualidade. Em relação ao acompanhamento farmacoterapêutico realizado pelo grupo, este permitiu uma visão social e humana completa do acadêmico frente aos pacientes beneficiados com o acompanhamento.

No ano de 2013, o NAFAU promoveu três Estudos Dirigidos em Atenção Farmacêutica (EDAF), três palestras, uma campanha de saúde no centro da cidade e um Workshop sobre adesão a terapia medicamentosa, atingindo, respectivamente, um público alvo de 80,113, 150 e 19 pessoas, totalizando 362, sendo estes diversificados em graduandos no Campus Sede da UNIFAL-MG e a população de Alfenas-MG. Além destas atividades, promoveu III Simpósio Internacional de Atenção Farmacêutica na UNIFAL-MG contando com a presença de ilustres palestrantes internacionais e nacionais da área clínica.

No ano de 2014, realizou-se 9 EDAF com temas relacionados à distúrbios menores e maiores, bem como saúde mental, com público de 125 graduandos. 4 palestras, com os temas: Fibromialgia, acupuntura, atenção farmacêutica em parasitoses e como cobrar pelos serviços farmacêuticos, sendo possível atingir 83 indivíduos. Organizou-se uma campanha de saúde de orientação em saúde em um supermercado local, com aferição de parâmetros bioquímicos e fisiológicos e orientação farmacêutica, sendo estes serviços destinados à 250 pessoas, além disso, promoveu 2 Workshops, sobre “ Uso correto de formas farmacêuticas" e "Workshop sobre adesão à terapia medicamentosa".

Em 2015, organizou-se o I Ciclo de Seminários do NAFAU com temas recorrentes à população acadêmica, frente à necessidade dos mesmos, sendo estes: Minicurso: Farmacoterapia das dislipidemias $(\mathrm{n}=20)$, Palestra: Dependência química $(n=45)$, Anticoncepcional oral e pílula do dia seguinte $(n=43)$, Depressão e suicídio $(\mathrm{n}=32)$, Tratamento farmacológico da dor $(n=33)$. A fim de promover a saúde, foram montados stands educativos com distribuição de folders informativos e são realizados os testes de colesterol, glicemia e aferição de pressão, em conjunto com a orientação farmacêutica. Realizou-se 5 campanhas de saúde para promoção do uso racional dos medicamentos para a comunidade externa e acadêmica, atingindo um público alvo de aproximadamente 1000 pessoas.

No ano corrente, foi promovido e ministrado por membros do grupo, o Estudo Dirigido em Atenção Farmacêutica em dois dias, com os temas: Saúde do homem, da mulher e do idoso, do qual se abordou as principais enfermidades, medicamentos mais utilizados e a atuação do farmacêutico frente a saúde dos mesmos. Os temas trabalhados pelo NAFAU são baseados na deficiência 
conceitual e prática dos graduandos da Universidade, para complementação da grade curricular dos cursos, bem como atualização dos conhecimentos já adquiridos.

Notada a demanda e a necessidade da população quanto a informações em saúde, orientações do uso racional de medicamentos e controle dos parâmetros bioquímicos e fisiológicos, o NAFAU realiza frequentemente campanhas de saúde. Assim, no presente ano, realizou-se 4 campanhas de saúde, tanto para a comunidade externa, quanto acadêmica, com enfoque no câncer de pele e sua prevenção; Dengue, Zika e Chikungunya; e orientações farmacêuticas em saúde,atingindo 1050 participantes. Foram realizados nessas ações aferição de pressão, glicemia, triglicérides e colesterol, além de distribuição de materiais educativos, espaço aberto para dúvidas e orientações aos envolvidos.Com o feedback oral recebido por parte da comunidade, observou-se um grande impacto social frente aos serviços prestados, uma vez que os mesmos são diminuídos e/ou escassos na cidade, podendo por vezes ser de difícil acesso.

De acordo com o CRF-SP, 2016, a falta de orientação é um dos principais obstáculos a prevenção de enfermidades e de suas complicações, acarretando um alto custo para o sistema público de saúde. A realização de campanhas de orientação além de ser um estímulo ao trabalho dos futuros farmacêuticos como profissionais e educadores em saúde, tendem a auxiliar o serviço público de saúde a minimizar custos com internações.

Segundo o IBGE (2015) apenas um terço da população brasileira possui planos de saúde e grande parcela da população tem dificuldades em ter acesso ao sistema de saúde e também a informações de saúde de qualidade. É importante ressaltar também a necessidade de que as ações extensionistas sejam realizadas de uma maneira acessível, aproximando a linguagem dos executores da ação à da população, utilizando termos de fácil entendimento e buscando sempre conhecer as necessidades e as demandas da população.

Considerando a abrangência virtual e o crescente uso de ferramentas online como fonte de ensino e informação, o núcleo possui um blog e um perfil na rede social Facebook, com atualização semanal, abordando notícias e atualidades na área da saúde, baseando-se embases científicas a fim de sanar dúvidas e difundir conhecimentos com assuntos pertinentes da área farmacêutica e clínica, sendo estas publicações supervisionadas e revisadas pela coordenadora do NAFAU. Desde a criação, janeiro de 2012, o blog teve 16.792 acessos e possui 1.222 amigos no perfil na rede social (Figura 1). 
Figura 1 - Levantamento do público atingido nos anos de 2012 a julho/2016.

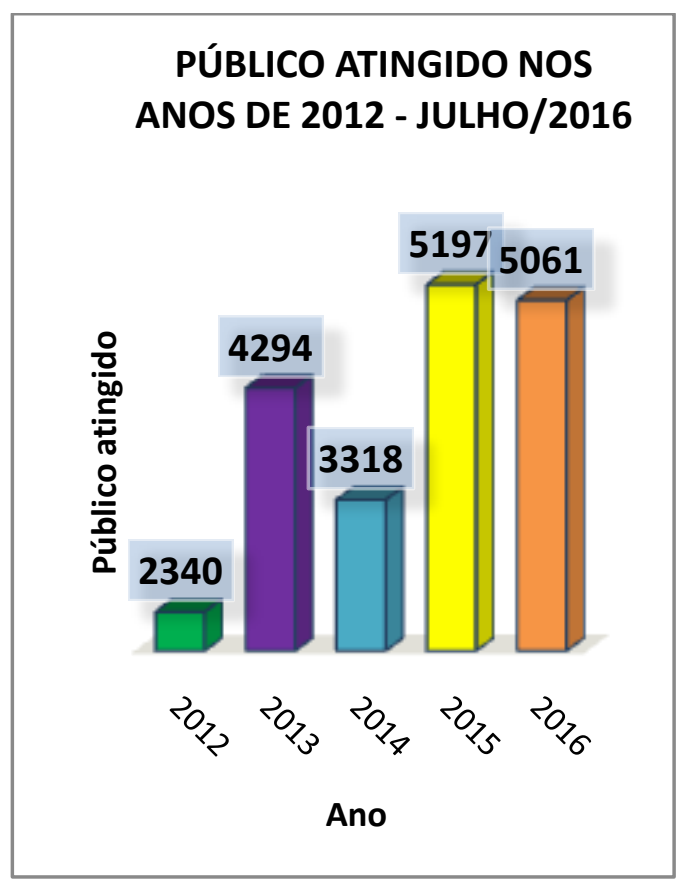

Fonte: Autores (2016).

Analisando os dados expostos nesse gráfico pode-se observar o aumento do alcance nas atividades do programa, uma vez que o público atingido até o período de julho do ano de 2016 está correspondendo quase ao valor total atingido no ano de 2015. Esses dados são explicados pelo planejamento de novas ações e intensificação das atividades.

O núcleo possui além do caráter de cunho social, a interface de pesquisa,com apresentação de trabalhos científicos em eventos regionais, nacionais e internacionais, como o Simpósio Internacional de Atenção Farmacêutica, Congresso Mineiro de Farmácia, Semana Farmacêutica da UNIFAL-MG, Congresso Integrado da UNIFAL, Encontro Farmacêutico de Ribeirão Preto e Congresso Brasileiro de
Extensão. Os trabalhos foram apresentados em formato de banner e apresentação oral. Dois trabalhos de pesquisa vinculados ao grupo receberam menções honrosas, sendo estes: Uso da telemedicina para acompanhamento farmacoterapêutico de pacientes com depressão - Encontro Farmacêutico de Ribeirão Preto e Effectiveness of pharmaceutical care in patients suffering from depression - IV Simpósio Internacional de Atenção Farmacêutica.

Com o projeto Atenção Farmacêutica no seu Lar (AtenFar), no qual é realizado o acompanhamento farmacoterapêutico dos pacientes, foi possível acompanhar 48 pacientes desde a sua criação. Cada membro do grupo realizou visitas domiciliares aos pacientes e nestas foram realizadas aferições de pressão arterial, glicemia capilar conforme a necessidade do paciente, intervenções farmacêuticas para identificação e solução dos problemas relacionados aos medicamentos e os pacientes eram orientados quanto a utilização correta dos medicamentos. Neste ano corrente, o projeto está em execução e há 15 pacientes assistidos.

Não é possível pensar em saúde sem pensar em educação e nas relações que existem entre ambas (GAZZINELLI, 2006). É nesse contexto que se insere a importância do farmacêutico como educador em saúde, capaz de atuar em defesa da saúde do 
usuário através da promoção do uso racional de medicamentos, bem como na participação do processo educativo dos pacientes no que diz respeito aos riscos da automedicação, das interações medicamentosas, manejo de efeitos indesejados e incentivo da adesão ao tratamento.

Ainda, o projeto também vinculado ao Núcleo, "Drogas: uma questão de escolha?" ministrou 3 palestras educativas no Centro de Referência de Assistência Social (CRAS) do Bairro Campos Elísio de Alfenas-MGe outras 17 para alunos de ensino médio na Escola Estadual Judith Viana, abordandoas drogas de uso mais comum e suas consequências ao organismo, visando a prevenção do uso, abuso e dependência de drogas ilícitas e lícitas. Durante a execução constatou-se à necessidade de orientação e a continuidade do projeto no local para a resolução do uso e abuso de drogas.

Os programas de extensão universitária são importantes devido a relação estabelecida entre a Universidade e a sociedade, sendo consolidada através da aproximação e troca de conhecimentos e experiências entre professores, alunos e população, possibilitando o desenvolvimento de processos de ensino e aprendizagem a partir de práticas cotidianas aliadas com 0 ensino e pesquisa e, especialmente, por propiciar o confronto da teoria com o mundo real de necessidades e desejos. $\mathrm{Na}$ área da saúde, tem-se uma importância particular na medida em que se integram à rede assistencial e destinam um espaço diferenciado para novas experiências voltadas à humanização, ao cuidado e à qualificação da atenção à saúde (HENNINGTON, 2005).

Em relação às dificuldades, o programa ainda não possui uma sala própria para o desenvolvimento de suas atividades uma vez que outros programas da Universidade possuem um local específico para suas reuniões e planejamento de ações. O programa não possui fomento para a sua realização no tocante à aquisição de material de custeio, os quais são necessários principalmente para a realização das campanhas de saúde, recebendo apenas uma bolsa da Pró-Reitoria de Extensão que é destinada a um aluno do grupo. Sendo assim, o grupo possui um único bolsista, sendo os demais participantes não bolsistasvoluntários.

\section{CONCLUSÃO}

O programa contribuiu para a multiplicação do conhecimento em saúde tanto para a equipe integrante quanto para a comunidade externa, utilizando também as redes sociais como ferramenta de informação. Ainda, devido sua interface em pesquisa e extensão, possui em desenvolvimento vários projetos que atuam 
em todas as faixas etárias e sociais, e

atualmente com deficientes visuais,

proporcionando a população informações e cuidado de qualidade. Enfatiza-se assim a extrema importância da extensão universitária, promovendo a saúde e aproximando o acadêmico da população e de suas reais necessidades e demandas.

\section{REFERÊNCIAS:}

BRASIL. Fascículo V: O percurso histórico da atenção farmacêutica no mundo e no Brasil. CRF - SP: Conselho Regional de Farmácia do Estado de São Paulo; Organização PanAmericana de Saúde. Brasília, 2010.

\section{CONSELHO REGINAL DE FARMÁCIA.}

Disponívelem:

$<$ http://portal.crfsp.org.br/index.php/campanhas. html>. Acesso: 30 de junho de 2016>

FUNDAÇÃO EZEQUIEL DIAS. A Assistência Farmacêutica na Atenção à Saúde. Edição revisada e ampliada. Belo Horizonte: Ed. FUNED, 144 p. ISBN: 978-85-99902-03-5. 2010.

GAZZINELLI, M. F; REIS, D. C.; MARQUES, R. C. Educação em saúde: teoria, método e imaginação. Belo Horizonte: Ed UFMG, 2006. $166 \mathrm{p}$.

HENNINGTON,É. A. Acolhimento como prática interdisciplinar num programa de extensão universitária Shelter as na interdisciplinary practice in a university extension program. Cad. Saúde Pública, Rio de Janeiro, 21(1):256-265, jan-fev, 2005

NOGUEIRA, Maria das Dores Pimentel(org.). Extensão universitária: diretrizes conceituais e políticas Belo Horizonte: Fórum Nacional de Pró-Reitores de Extensão das Universidades Públicas/ UFMG, 2000. 193 p.

OLIVEIRA, A.B.; OYAKAWA, C.N.; MIGUEL, M.D.; ZANIN, S.M.W.; MONTRUCCHIO, D.P. Obstáculos da Atenção Farmacêutica no Brasil. Revista Brasileira de Ciências Farmacêuticas, v.41, n.4, p.409-413, 2005.
OLIVEIRA, B. A.; OYAKAWA, C. N.; MIGUEL, M. D.; ZANIN, S. M. W.; MONTRUCCHIO, D. P. Obstáculos da atenção farmacêutica no Brasil. Revista Brasileira de Ciências Farmacêuticas Brazilian Journal of Pharmaceutical Sciences. vol. 41, n. 4, out./dez., 2005.

PEREIRA, L. R. L.; FREITAS, O. A evolução da Atenção Farmacêutica e a perspectiva para o Brasil. Revista Brasileira de Ciências Farmacêuticas. vol. 44, n. 4, out./dez., 2008.

PEREIRA, L. R. L; FREITAS, DE O. A evolução da Atenção Farmacêutica e a perspectiva para o Brasil. Revista Brasileira de Ciências Farmacêuticas. Brazilian Journal ofP harmaceutical Sciences. vol. 44, n. 4, out./dez., 2008.

STRAND, L.M.; CIPOLLE, R.J.; MORLEY, P.C.; FRAKES, M.J. The impact of pharmaceutical care practice on the practitioner and the patient in the ambulatory practice setting: twenty five years of experience. Curr. Pharm. Des., v.10, n.31, p.3987-4001, 2004.

UFMG (Universidade Federal de Minas Gerais) RENEX: Rede Nacional de Extensão Universitária. Disponível em <renex.org.br>.

\section{Agradecimento aos parceiros}

- Pró-Reitoria de Extensão da Universidade Federal de Alfenas.

- Equipe integrante do NAFAU. 